This is final peer-reviewed manuscript accepted for publication in Journal of Drug Delivery Science and Technology 36, 208-215, 2016, DOI: 10.1016/j.jddst.2016.10.011

\title{
Improving Ibuprofen solubility by surfactant-facilitated self-assembly into mixed micelles
}

\author{
Katerina Stoyanova ${ }^{\mathrm{a}}$, Zahari Vinarov ${ }^{* a}$, Slavka Tcholakova ${ }^{\mathrm{a}}$ \\ ${ }^{a}$ Department of Chemical \& Pharmaceutical Engineering, Faculty of Chemistry \& Pharmacy, \\ Sofia University, 1164 Sofia, Bulgaria
}

\section{*Corresponding author:}

Assist. Prof. Zahari Vinarov

Department of Chemical and Pharmaceutical Engineering

Faculty of Chemistry and Pharmacy, Sofia University

1 James Bourchier Ave., 1164 Sofia

Bulgaria

Phone: (+359-2) 9625310

Fax: (+359-2) 9625643

E-mail: ZV@LCPE.UNI-SOFIA.BG

Running head: Surfactant-facilitated self-assembly of Ibuprofen into mixed micelles 


\begin{abstract}
Ibuprofen is a poorly water-soluble drug, characterized by dissolution-limited oral bioavailability. One approach to improve its water solubility and bioavailability is by solubilizing it in micellar surfactant solutions. Here we investigate the effect of the surfactant type and the mechanism of solubility enhancement of Ibuprofen in surfactant solutions. The equilibrium Ibuprofen solubility in solutions of six surfactants was determined by HPLC. The nonionic surfactant polysorbate 80 (Tween 80 ), and the anionic surfactants sodium dodecyl sulfate (SDS) and sodium lauryl ethoxy (3) sulfate (SLES-3EO) improve the Ibuprofen solubility by a factor of 200 , as compared to the solubility in water. The highest Ibuprofen solubility is observed in SDS and SLES-3EO solutions, containing $0.6 \mathrm{M} \mathrm{NaCl}$. The mole fraction of Ibuprofen in the micelles and the transfer energy of Ibuprofen molecules from the aqueous phase into the micelle environment were determined by thermodynamic analysis of the solubility data. The maximum Ibuprofen mole fraction in the micelles of all studied surfactants is exceptionally high (between 0.4 and 0.6). Thus we can conclude that the main mechanism of Ibuprofen solubility enhancement is a selfassembly within mixed micelles with the main surfactant. The energy of co-micellization is estimated to be around $14 k T$ per Ibuprofen molecule.
\end{abstract}

Keywords: Ibuprofen, poorly water-soluble drugs, mixed micelles, solubilization, oral bioavailability. 


\section{INTRODUCTION}

More than $40 \%$ of the new chemical entities that emerge from modern drug discovery programs are characterized by poor water solubility [1]. The slow and incomplete dissolution of such drugs in the gastro-intestinal fluids limits their oral bioavailability and presents a significant problem in drug development. One of the classical approaches to improve the water solubility of hydrophobic drugs, which is still being used in the pharmaceutical industry, is to use appropriate surfactants [2-5].

Surfactants are a large group of pharmaceutical excipients, which are used in a variety of drug delivery vehicles as solubilizers, emulsifiers, foamers, wetting agents, etc. [6]. Above the critical micelle concentration (CMC) the surfactant molecules form micelles [7]: molecular aggregates which have a hydrophobic core and a hydrophilic surface. The hydrophobic interior of the micelles provides a suitable environment for hydrophobic molecules, which leads to the solubilization phenomenon [6-8], namely, a significant increase of the solubility of poorly water-soluble molecules in the micellar solutions, due to their incorporation in the surfactant micelles. On the other hand, amphiphilic drugs like nortriptyline hydrochloride and promazine hydrochloride can form mixed micelles with the classical surfactants $[9,10]$ which also leads to a strong enhancement of their solubility.

In the current article we investigate the effect of surfactants on the solubility of the non-steroidal anti-inflammatory drug Ibuprofen (IBP), which is used to relieve pain, fever and inflammation. IBP is a weak acid with $\mathrm{pKa} \approx 4.4$, solubility in water around $11 \mu \mathrm{g} / \mathrm{mL}$, and high membrane permeability [11]. Since the IBP molecule can ionize, its solubility depends strongly on the solution $\mathrm{pH}$. Thus, IBP is poorly soluble in the stomach, where $\mathrm{pH}$ ranges between 2 and 3 [12], whereas its solubility increases significantly in the small intestine (pH between 4.5 and 7.5 [13]). For example, IBP solubility at $\mathrm{pH} 5$ and 7.5 is 140 and $2300 \mu \mathrm{g} / \mathrm{mL}$, respectively [14].

However, the drug solubility in water per se does not provide direct information whether the drug will be sufficiently soluble in the gastro-intestinal tract. The orally administered drug dose differs strongly, depending on the drug type and the therapeutical application. The Biopharmaceutical Classification System (BCS), introduced by Amidon et al. [15], defines the so-called "dose number" (Do), which takes into account both the drug dose per given volume and the equilibrium drug solubility. Thus, drugs for which Do $\leq 1$ are classified as highly soluble, whereas all others have poor water solubility [16].

The maximum single dose of IBP is relatively high $(800 \mathrm{mg})$ and for this reason IBP's Do is always bigger than one: $\mathrm{Do}=290$ at $\mathrm{pH}=2$ (approximate conditions in the stomach) and $\mathrm{Do}=1.4$ at $\mathrm{pH}=$ 7.5 (maximum $\mathrm{pH}$ value in the small intestine). Thus we see that the IBP concentration in the stomach fluids, after oral administration of $800 \mathrm{mg}$ IBP, is 290 times higher than the equilibrium IBP solubility under these conditions. As a result, IBP is characterized by poor water solubility and is classified as Class II drug according to BCS [17].

There are different approaches for enhancing IBP delivery: controlled-release formulations [1820], lipid-based drug delivery systems [21-23], nano-particles [24,25], vesicles [26] or solubilization by surfactants [27-29].

Surfactants are reported to increase significantly IBP solubility [27-29] and are thus expected to improve its oral bioavailability. The effect of the concentration of sodium dodecyl sulfate (SDS), dodecyl octa(ethylene oxide) (C12E8) and dodecyltrimethyl-ammonium bromide (DTAB) on the IBP solubilization at $\mathrm{pH} 7.4$ was studied by Stephenson et al. [27]. These authors found that the aqueous 
solubility of IBP increases linearly with surfactant concentration for these surfactants. The highest solubilization was observed upon the addition of $\mathrm{DTAB}$, followed by $\mathrm{C}_{12} \mathrm{E}_{8}$ and SDS. A molecularthermodynamic modeling approach was developed to predict theoretically the solubilization behavior of these systems. The obtained theoretical results on the IBP solubility in SDS and $\mathrm{C}_{12} \mathrm{E}_{8}$ solutions were in a good agreement with the experimental data.

Kokot \& Zmidzinska [28] studied the IBP solubilization in unbuffered SDS, Brij 35 and Tween 60 surfactant solutions. They reported a significant increase of IBP solubility and no specific effect of the surfactant type.

Park et al. studied the saturation solubility of IBP [29] and showed that at $\mathrm{pH}=1.2$, highest solubility is obtained with solutions of cetyltrimethylammonium bromide (CTAB), compared to much lower solubility for Tween 80 and SDS. The better IBP solubilization in CTAB, compared to SDS solutions, was explained by attractive electrostatic interactions, without accounting for the longer hydrophobic chain length of CTAB. The authors reported also a higher dissolution rate of IBP tablets in surfactant solutions, relative to pure water.

None of the above studies has provided mechanistic explanation for the observed very strong effects of surfactants on IBP solubility. Therefore, the aim of the current article is to clarify (1) the mechanism of IBP solubility enhancement in surfactant solutions and (2) the effect of the surfactant type. To achieve this aim we determined experimentally the effect of four nonionic (Tween 20, 40, 60 and 80) and two anionic (SDS and SLES-3EO) surfactants on the IBP solubility. The mechanism of improved IBP solubility and the strength of the drug-surfactant interactions are analyzed using a thermodynamic treatment of the solubility data.

\section{MATERIALS AND METHODS}

\subsection{Materials.}

\subsubsection{Drug and surfactants.}

We used IBP (see Figure 1), product of Sigma Aldrich $\left(M_{\mathrm{W}}=206.29 \mathrm{~g} / \mathrm{mol}\right.$, purity $99 \%$, cat. no. I4883). To increase the drug solubility, we used several nonionic and anionic surfactants. Table 1 provides information about all studied surfactants: type, trade name/abbreviation used in the text, purity, molecular weight, chemical formula, producer, and critical micelle concentration (CMC). The molecular structures of the studied surfactants are presented in Figure 2.

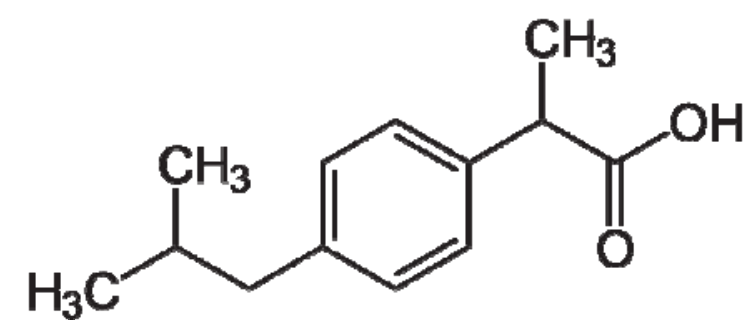

Figure 1. Molecular structure of IBP. 
Table 1. Studied surfactants.

\begin{tabular}{|l|l|l|l|l|l|}
\hline $\begin{array}{l}\text { Type of } \\
\text { surfactant }\end{array}$ & $\begin{array}{l}\text { Trade name/ } \\
\text { abbreviation }\end{array}$ & $\begin{array}{l}\text { Purity, } \\
\text { wt\% }\end{array}$ & $\begin{array}{l}\text { Molecular } \\
\text { mass, g/mol }\end{array}$ & Producer & CMC, mM \\
\hline \multirow{3}{*}{ Nonionic } & Tween 20 & 100 & 1228 & Sigma & $0.08[30]$ \\
\cline { 2 - 6 } & Tween 40 & 100 & 1277 & Sigma & $0.027[31]$ \\
\cline { 2 - 6 } & Tween 60 & 100 & 1309 & Sigma & $0.017[31]$ \\
\cline { 2 - 6 } & Tween 80 & 100 & 1310 & Sigma & $0.023[31]$ \\
\hline \multirow{2}{*}{ Anionic } & SDS & 99 & 288 & Acros & $8.0[32]$ \\
\cline { 2 - 6 } & SLES 3 EO & 70 & 420 & Stepan Co. & $0.5[33]$ \\
\hline
\end{tabular}

\section{Tween 20}

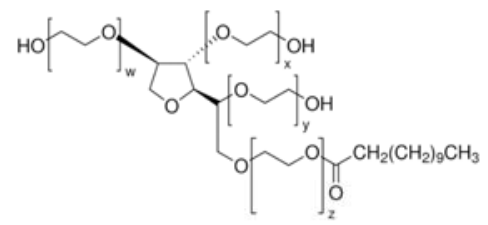

Figure 2. Molecular structures of the studied surfactants.
Tween 40

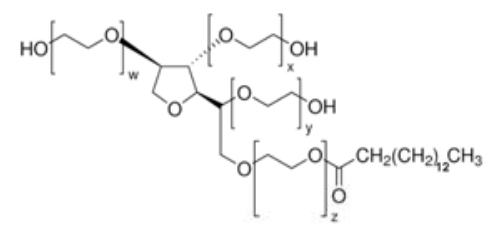

Tween 80
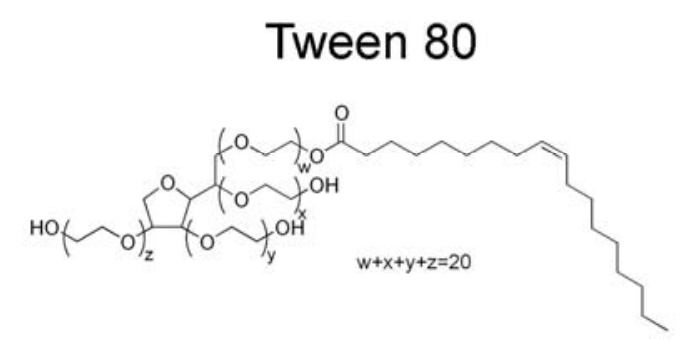

Tween 60

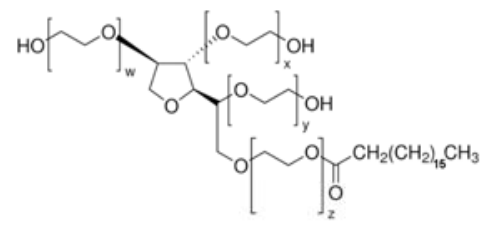

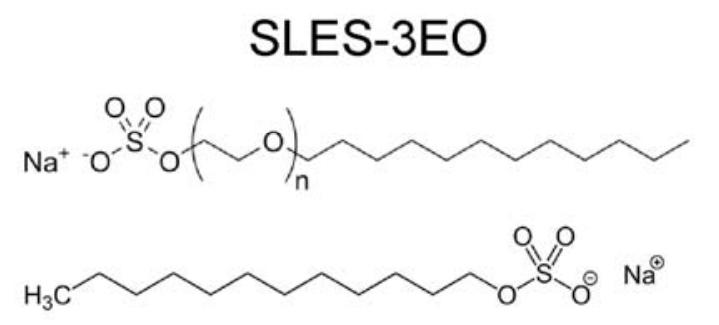

\section{SDS}




\subsubsection{Buffer solutions, solvents for HPLC and water.}

To prepare the buffer solutions we used $\mathrm{H}_{3} \mathrm{PO}_{4}\left(85 \%\right.$, Merck, cat. no. 100563), $\mathrm{NaH}_{2} \mathrm{PO}_{4}(99 \%$, Fluka Analytical, cat. no. 71504), $\mathrm{Na}_{2} \mathrm{HPO}_{4} .7 \mathrm{H}_{2} \mathrm{O}$ (99 \%, Riedel de Haën, cat. no. 30413), $\mathrm{CH}_{3} \mathrm{COOH}$ (100\%, Merck, cat. no. 100056) and $\mathrm{CH}_{3} \mathrm{COONa} .3 \mathrm{H}_{2} \mathrm{O}(99 \%$, Merck, cat. no. 106267).

The mobile phase solvents for HPLC analysis include acetonitrile (HPLC grade, $99 \%$ ) and $20 \mathrm{mM}$ aqueous solutions of $\mathrm{CH}_{3} \mathrm{COOH}$ and $\mathrm{CH}_{3} \mathrm{COONa}$. All aqueous solutions and buffers were prepared using deionized water from water-purification system Elix 3 (Millipore, USA).

\subsection{Methods.}

\subsubsection{Determination of equilibrium solubility of IBP.}

We determined the effect of the $\mathrm{pH}$ on the IBP solubility in aqueous phase using the following procedure: we weighed $20 \mathrm{mg}$ IBP in a $20 \mathrm{~mL}$ bottle and then added $10 \mathrm{~mL}$ buffer solution with a $\mathrm{pH}$ value in the range between 3.5 and 6 . The mixture was then stirred on a magnetic stirrer for $24 \mathrm{~h}$, at 400 rpm and $37^{\circ} \mathrm{C}$.

The effect of surfactants was studied at a constant surfactant concentration of $0.5 \mathrm{wt} \%$. We first prepared $10 \mathrm{~mL}$ of $4 \mathrm{wt} \%$ surfactant solution; then, we weighed $20 \mathrm{mg}$ IBP in another bottle of $20 \mathrm{~mL}$ and added $1.25 \mathrm{~mL}$ of the respective $4 \mathrm{wt} \%$ surfactant solution and $8.75 \mathrm{~mL}$ water. For the experiments in the presence of $600 \mathrm{mM} \mathrm{NaCl}$ we dissolved the surfactant in a freshly prepared $600 \mathrm{mM} \mathrm{NaCl}$ solution which was used also for dilution in the mixtures of surfactant and IBP. We prepared similarly the solutions for the experiments performed in the presence of buffer.

All mixtures were stirred for $24 \mathrm{~h}$ with a magnetic stirrer at $400 \mathrm{rpm}$ and $37^{\circ} \mathrm{C}$. After incubation, the obtained IBP suspension was filtered through $200 \mathrm{~nm}$ NYLON syringe filter (thermostated at $37^{\circ} \mathrm{C}$ ) to eliminate the undissolved particles. Finally, the concentration of the dissolved drug in the obtained clear filtrate was determined by HPLC. The samples temperature was maintained at $37^{\circ} \mathrm{C}$ during all stages of this procedure.

\subsubsection{HPLC analysis.}

The HPLC analysis was carried out on a Shimadzu apparatus, equipped with two high-pressure mixing binary gradient pumps (LC-20AD), autosampler (SIL-10ADvp), four-line membrane degasser (DGU-14A), wide temperature range column oven (CTO-10ASvp) and a dual-wave length UV-VIS detector (SPD-10Avp).

We modified an analytical procedure, described in the United States Pharmacopoeia (USP). We used an XBridge C18 column $\left(100 \times 4.6 \mathrm{~mm}^{2}, 3.5 \mu \mathrm{m}\right.$ particle size $)$ and an isocratic elution for 10 min with total flow of $1 \mathrm{~mL} / \mathrm{min}$, with a mobile phase of acetic buffer $(\mathrm{pH}=4)$ and acetonitrile with 40:60 vol:vol ratio. UV detection was performed at $\lambda=214$ and $254 \mathrm{~nm}$. Column temperature was set at $40{ }^{\circ} \mathrm{C}$.

The retention time of IBP was $t_{\mathrm{R}}=4.6 \mathrm{~min}$ for all conditions studied. The concentration of soluble drug was determined by using a standard curve, which was prepared by dissolving a known amount of drug in a buffer solution with pH 6.5 (see Figure 3). We used the slope of the curve to calculate the IBP concentration in all studied solutions, according to the equation: 
A chromatogram from the HPLC-analysis of IBP is presented in Figure 4.

Ibuprofen in Buffer Solution, $\mathrm{pH}=6.5$ Calibration Curve

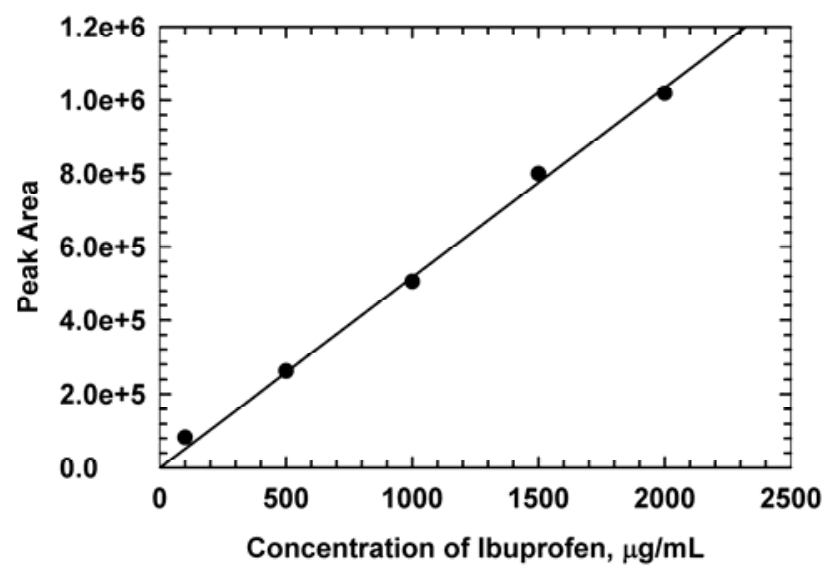

Figure 3. Standard curve of IBP in buffer solution with $\mathrm{pH}=6.5$.

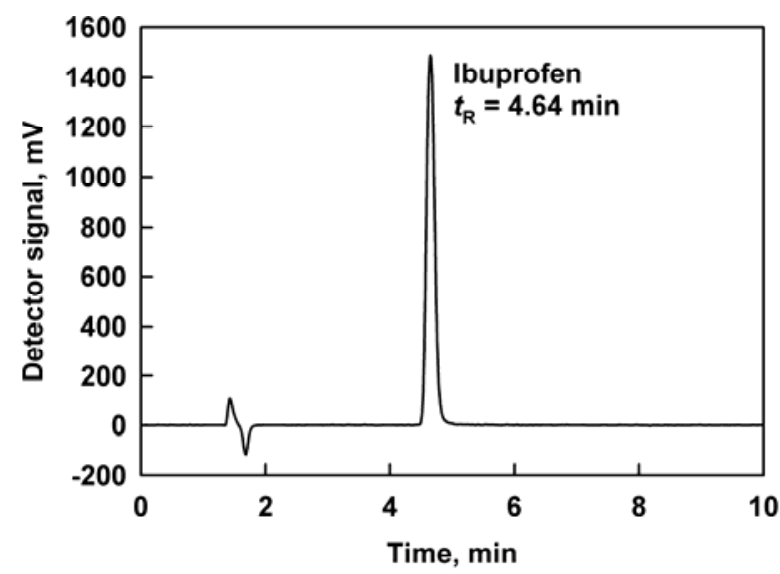

Figure 4. Representative HPLC chromatogram of $1 \mathrm{mg} / \mathrm{mL}$ IBP standard solution, prepared in $\mathrm{pH}=6.5$ buffer.

\subsubsection{Solubility enhancement.}

To determine and compare the effect of the studied surfactants on the IBP solubility, we expressed the results in terms of the so-called "solubility enhancement", calculated from the ratio [drug solubility in surfactant solution $\left.\left(S_{\text {tot }}\right)\right]$ / [drug solubility in water $\left.\left(S_{\mathrm{w}}\right)\right]$ :

Solubility enhancement $=S_{\mathrm{tot}} / S_{\mathrm{W}}$ 
In our study, we obtained a value of $7 \mu \mathrm{g} / \mathrm{mL}$ for the intrinsic solubility of IBP in water (no buffer), which is in a reasonable agreement with literature values $\left(S_{\mathrm{W}}=10 \mu \mathrm{g} / \mathrm{mL}[32]\right)$.

\section{Results and discussion}

In section 3.1 we describe the dependence of IBP solubility on $\mathrm{pH}$. In section 3.2 we present the effect of all studied surfactants on the equilibrium solubility of IBP. In section 3.3 we analyze the obtained experimental results using a thermodynamic approach.

\subsection{IBP solubility dependence on pH.}

IBP is a weak acid ( $\mathrm{pKa}=4.4)$ and its solubility depends strongly on $\mathrm{pH}$. To differentiate clearly the effects of the surfactants from those of $\mathrm{pH}$, in the first series of experiments we determined the IBP solubility in different buffer solutions (without surfactant) using the procedure from section 2.2.1.

The obtained results are presented in Figure 5. We observed very low IBP solubility at acidic pH $(\mathrm{pH} \leq 4)$. This makes IBP practically insoluble in the $\mathrm{pH}$ range, characterizing the stomach [12]. At $\mathrm{pH} \geq$ 4.5 the solubility increases and it becomes $920 \mu \mathrm{g} / \mathrm{mL}$ at $\mathrm{pH}=6$. Values of this range could be observed in the small intestine [13]. These results are in a good agreement with the pKa value of IBP and with the results presented by Yazdanian et al. [14], viz. $140 \mu \mathrm{g} / \mathrm{mL}$ at $\mathrm{pH}=5$ [14], compared to $170 \mu \mathrm{g} / \mathrm{mL}$ in the current study. The slightly higher solubility determined in our study could be explained with the higher temperature in our experiments, $37^{\circ} \mathrm{C}$, compared to $25^{\circ} \mathrm{C}$ in [14].

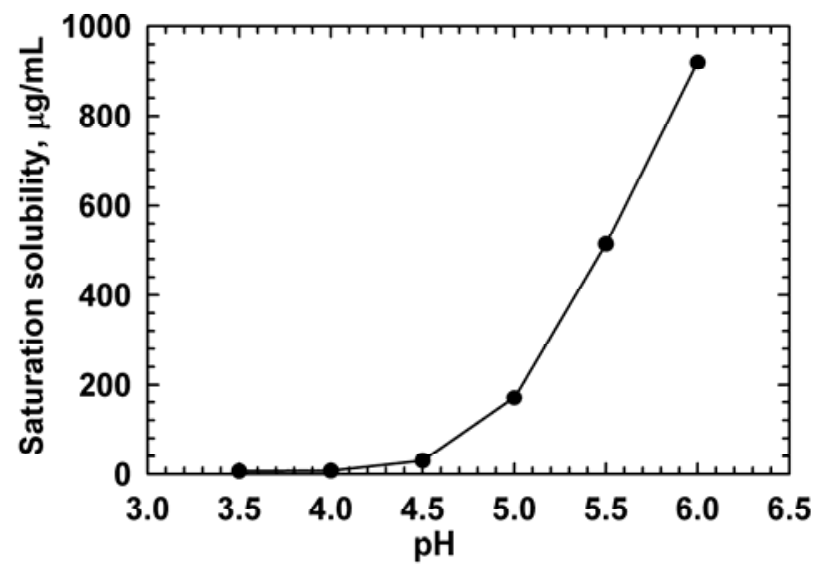

Figure 5. Effect of $\mathrm{pH}$ on the equilibrium solubility of IBP.

\subsection{Effect of the presence of surfactant on the equilibrium solubility of IBP.}

In this section we present the results for the solubility of IBP in aqueous solutions of different surfactants in unbuffered solutions. The experiments were carried out at a constant surfactant 
concentration $0.5 \mathrm{wt} \%$. The solutions were prepared using the procedure from section 2.2 .1 and the concentration of the dissolved drug was measured after $24 \mathrm{~h}$ stirring at $37^{\circ} \mathrm{C}$ (equilibrium solubilization).

The $\mathrm{pH}$ values of the surfactant + IBP mixtures were measured in the end of the experiment and were close for all studied micellar solutions (between 3.5 and 4.1), despite the differences in the initial $\mathrm{pH}$ (see Table 2). An exception of this rule is SLES-3EO, for which the initial and the final $\mathrm{pH}$ were 9.80 and 4.85 , respectively. In most cases, $\mathrm{pH}$ in the end of the experiment was lower than its initial value, most probably due to deprotonation of some fraction of the IBP molecules. The observed low values of $\mathrm{pH}$ cannot be explained with the dissolution of $\mathrm{CO}_{2}$ from the air because, at a normal partial pressure of $\mathrm{CO}_{2}$ $\left(3.5 \times 10^{-4} \mathrm{~atm}\right)$, the dissolved $\mathrm{CO}_{2}$ maintains $\mathrm{pH} \approx 5.6$. To obtain $\mathrm{pH} \approx 4$, the required $\mathrm{CO}_{2}$ pressure should be $p\left[\mathrm{CO}_{2}\right] \approx 1 \mathrm{~atm}$ which is not the case in our experiments. Since the IBP solubility does not depend on the $\mathrm{pH}$ at values lower than 4.5 (see section 3.1), the observed variations in the drug solubility in these surfactant solutions are due only to the presence of surfactant micelles. Exception is SLES-3EO, for which the effect could be partly related to the higher values of the initial and the final $\mathrm{pH}$.

The experimental results for the IBP solubility in the solutions of all studied nonionic and anionic surfactants are compared in Figure 6. They show that the solubility of IBP, $S_{\text {tot, }}$ increases significantly in the presence of all surfactants studied. Most effective is Tween 80 , for which we observe IBP solubility of about $1400 \mu \mathrm{g} / \mathrm{mL}$, see Figure 6A, followed by Tween 40 and 60 with solubility of about 1200 and 1100 $\mu \mathrm{g} / \mathrm{mL}$, respectively. Tween 20 is the least effective nonionic surfactant with $S_{\text {tot }} \approx 900 \mu \mathrm{g} / \mathrm{mL}$.

Table 2. $\mathrm{pH}$ at the end of the experiment for $0.5 \mathrm{wt} \%$ surfactant solutions, in the absence and in the presence of IBP.

\begin{tabular}{|l|l|l|}
\hline Surfactant & $\begin{array}{l}\text { pH without } \\
\text { IBP }\end{array}$ & $\begin{array}{l}\text { pH in } \\
\text { presence of } \\
\text { IBP }\end{array}$ \\
\hline Tween 20 & 4.00 & 4.14 \\
\hline Tween 40 & 4.02 & 3.93 \\
\hline Tween 60 & 3.91 & 3.70 \\
\hline Tween 80 & 4.90 & 4.09 \\
\hline SDS & 3.65 & 3.49 \\
\hline SLES-3EO & 9.80 & 4.85 \\
\hline
\end{tabular}

In the absence of additional electrolytes (buffers or $\mathrm{NaCl}$ ), the anionic surfactants SDS and SLES3EO have similar effect, $S_{\text {tot }} \approx 1300 \mu \mathrm{g} / \mathrm{mL}$, close to that of Tween 80 . The addition of a relatively high concentration of electrolyte $(0.6 \mathrm{M} \mathrm{NaCl})$ increases further the IBP solubility, $S_{\text {tot }} \approx 1900 \mu \mathrm{g} / \mathrm{mL}$. We must note that this solubility is very close to the total IBP amount, added in the beginning of the experiment $(2000 \mu \mathrm{g} / \mathrm{mL})$. Therefore, the solubility of IBP in the presence of anionic surfactants, at high $\mathrm{NaCl}$ concentrations, could be even higher. 
Figure 6B compares the solubility enhancement of IBP in the studied surfactant solutions. Tween 80 and the nonionic surfactants (without electrolyte) increase the IBP solubility by a factor of 200, whereas Tween 20 - by a factor of 130. In the presence of electrolyte, the anionic surfactants enhanced the IBP solubility by a factor of 280 .
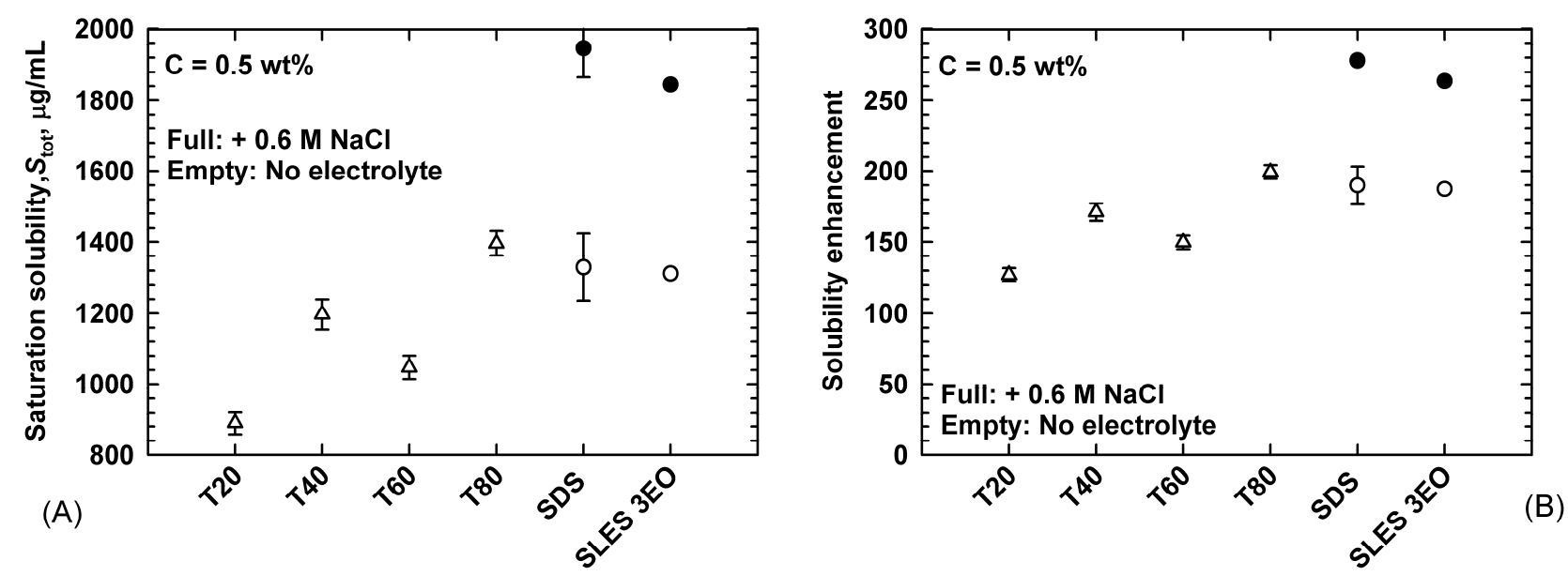

Figure 6. Effect of the type of nonionic (triangles) and anionic (circles) surfactants on (A) the equilibrium solubility and (B) the solubility enhancement of IBP. The experiments with the anionic surfactants are carried out in the presence (full circles) and in the absence (empty circles) of $0.6 \mathrm{M} \mathrm{NaCl}$. All experiments are performed at least in duplicate.

Table 3. Molar concentrations of the studied surfactants and solubility of IBP in their solutions. The solubility of IBP in water is $0.03 \mathrm{mM}$ at $37^{\circ} \mathrm{C}$.

\begin{tabular}{|l|l|l|}
\hline Surfactant type & Surfactant conc., mM & Dissolved IBP, mM \\
\hline Tween 20 & 4.07 & 4.20 \\
\hline Tween 40 & 3.91 & 5.66 \\
\hline Tween 60 & 3.82 & 5.19 \\
\hline Tween 80 & 3.82 & 6.65 \\
\hline SDS & 17.36 & 7.77 \\
\hline SDS + NaCl & 17.36 & 9.43 \\
\hline SLES-3EO & 11.91 & 7.31 \\
\hline SLES-3EO + NaCl & 11.91 & 8.94 \\
\hline
\end{tabular}

Page 10 of 19 
These results demonstrate the significant increase of the IBP solubility at fixed weight concentration of the surfactants studied. To investigate the molecular mechanism of the observed solubility increase, we calculated the molar concentrations of the surfactants and the dissolved IBP (see Table 3 above). One sees that the molar concentration of the solubilized IBP is comparable to the surfactant molar concentration and, in some cases, even higher. Thus, the obtained results show that the IBP mole fraction in the micelles is very high.

\subsection{Mole fraction and energy of incorporation of IBP in the mixed micelles.}

\section{(A) Mole fraction of IBP in the mixed micelles.}

To calculate the IBP mole fraction in the mixed micelles, $y_{\mathrm{d}}$, we used the following equation:

$$
y_{d}=\frac{S_{t o t}-S_{W}}{\left(C_{S}-C M C\right)+\left(S_{t o t}-S_{W}\right)}
$$

where $S_{\text {tot }}$ is the total drug solubility in the surfactant solution, $S_{\mathrm{W}}$ is the intrinsic water solubility of IBP, $C \mathrm{~s}$ is the surfactant concentration, and $\mathrm{CMC}$ is the respective critical micelle concentration. The subtraction of $S_{\mathrm{W}}$ and $\mathrm{CMC}$ from $S_{\text {tot }}$ and $C_{\mathrm{S}}$, respectively, allows us to exclude those IBP and surfactant molecules which are not incorporated in the mixed micelles. In writing eq. 3 we assume that the presence of IBP in the solution does not change the CMC of the respective surfactant (Table 1). All surfactants, except SDS, have been studied at much higher concentrations than their CMC ( $C$ s $>>C M C$ ) and, hence, a possible change in the assumed CMC value would have a very small effect on the obtained results for $y_{\mathrm{d}}$.

The results for $y_{\mathrm{d}}$ are presented in Figure 7. One sees that the mole fraction of IBP in the micelles of all studied surfactants is very high, $y_{\mathrm{d}}=0.4$ to 0.6 , which is possible only if mixed micelles are formed between the molecules of the main surfactant and the IBP. This conclusion is in agreement with the results from other studies [35] which showed that IBP is a surface active drug which is able to form micelles at high concentrations even in the absence of surfactants.

However, most other studies interpret the observed increase of IBP solubility as solubilization by surfactant micelles [28,29]. Exception is the study of Stephenson et al. [27], in which the authors accounted for the amphiphilic nature of IBP by treating it like a conventional surfactant in the proposed molecular-thermodynamic modelling approach. There are several main differences between the solubilization and co-micellization (formation of mixed micelles) mechanisms [7]. In the solubilization mechanism, the mole fraction of solubilized drugs is usually very small and the main properties of the micelles are governed by the surfactant molecules [4]. In contrast, the formation of mixed micelles implies a comparable mole fraction of the main surfactant molecules and the drug molecules [36], just as we observed with IBP. An important consequence of the different mechanisms of solubility enhancement is the kinetics of the process: the solubilization is usually very slow (time scale of hours and days [37]), whereas the formation of mixed micelles is a rapid process (time scale of seconds and minutes, similarly to the micellization of common surfactants [6,7]). Indeed, measurement of solubilization kinetics of IBP 
in $0.5 \mathrm{wt} \%$ solution of Tween 20 showed that drug the solubility increases very quickly: $840 \mu \mathrm{g} / \mathrm{mL}$ for 5 min, compared to the equilibrium solubility of $890 \mu \mathrm{g} / \mathrm{mL}$. The formation of mixed micelles between IBP and surfactants is supported also by Rub et al. [38], who studied in detail the properties of these drugsurfactant micelles. Therefore, we can expect that IBP will have very high solubilization rate in the presence of surfactants, even in the acidic medium of the stomach which should significantly shorten the plasma $t_{\max }$ values and improve the oral bioavailability.

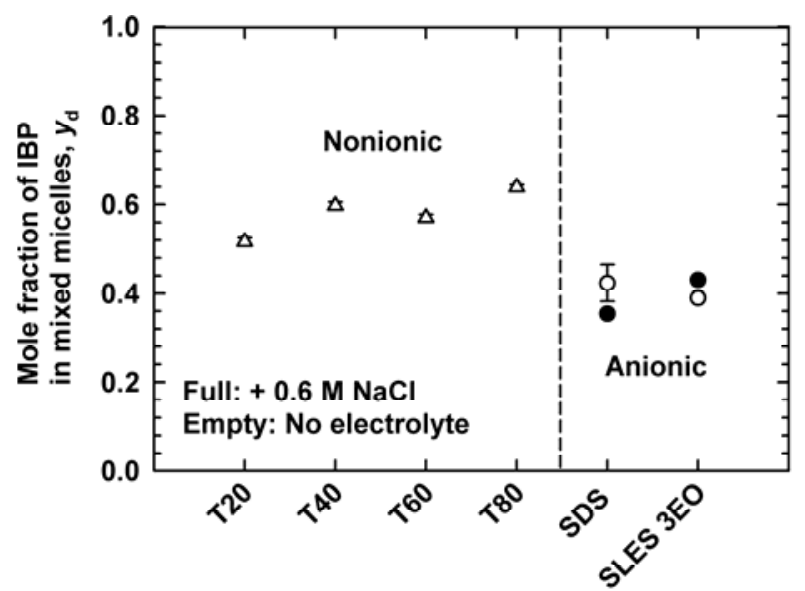

Figure 7. Mole fraction of IBP in the mixed micelles of all studied nonionic (triangles) and anionic (circles) surfactants, calculated using equation (3) above.

One can notice that the mole fraction of IBP in the micelles of the nonionic surfactants $\left(y_{\mathrm{d}} \approx 0.6\right)$ is higher than the one in the micelles of the anionic surfactants $\left(y_{\mathrm{d}} \approx 0.4\right)$, while the solubility enhancement in SDS and Tween 80 solutions is almost the same. This difference comes from the definition of $y_{\mathrm{d}}$, which includes the molar concentrations, instead of the weight concentrations. The molecular masses of the nonionic surfactants studied here are larger, compared to those of the anionic ones (e.g. $1310 \mathrm{~g} / \mathrm{mol} \mathrm{for}$ Tween 80, compared to $288 \mathrm{~g} / \mathrm{mol}$ for SDS). As a result, the molecular concentration in the nonionic surfactant solutions is much lower than in the anionic surfactant solutions, at the same weight concentration of surfactant. In other words, a solution of nonionic surfactants will contain significantly smaller number of micelles than a solution of anionic surfactant with equivalent weight concentration, considering the similar micelle aggregation numbers for these surfactants: $N_{\mathrm{agg}}=48$ to 75 for SDS [33] and $N_{\mathrm{agg}}=50$ for Tween 20 [39]. Thus, the concentration of solubilized IBP in SDS solutions is higher, compared to the nonionic surfactants (Table 3), because of the presence of more micelles in the SDS solutions. Note that IBP molecules are not charged under these experimental conditions $(\mathrm{pH}<4.5)$ and, hence, electrostatic repulsion between IBP and the anionic surfactant molecules is not expected.

As the nonionic surfactants solubilize significant concentration of IBP (Table 3) in much smaller number of micelles, the mole fraction of IBP in the micelles of nonionic surfactants is higher than the one in the micelles of anionic surfactants. The highest IBP mole fraction was determined in the micelles of Tween $80, y_{\mathrm{d}}=0.64$, i.e. the IBP molecules represent almost $2 / 3$ of all molecules in these mixed micelles. 
In the micelles of Tween 40 and 60 the mole fraction of IBP is lower $\left(y_{\mathrm{d}}=0.58\right)$. The micelles of Tween 20 contain the smallest fraction of IBP molecules $\left(y_{\mathrm{d}}=0.51\right)$.

The mole fraction of IBP in the anionic surfactants SDS and SLES 3-EO is lower compared to the one we obtained in all nonionic surfactants, $y_{\mathrm{d}} \approx 0.4$. The addition of electrolyte does not change $y_{\mathrm{d}}$ despite the observed increase in drug solubility. The presence of $0.6 \mathrm{M} \mathrm{NaCl}$ screens the electrostatic repulsion between the charged head groups of the ionic surfactants and results in decrease of the CMC which, in turn, increases the fraction of surfactant molecules forming micellar aggregates (vs. the free surfactant monomers). Thus, the main effect of the $\mathrm{NaCl}$ is the decrease the $\mathrm{CMC}$ of the anionic surfactant.

Let us now examine the relation between the molecular structure of the surfactants and the incorporation of IBP into the mixed micelles. For this aim we compare the IBP mole fraction in the micelles of surfactants with the same hydrophobic chain length but different hydrophilic head, and vice versa (surfactants with same heads but different chain lengths). First, we compare the surfactants with the same chain length (C12): SDS, SLES-3EO and Tween 20. The addition of an ethoxy group to the dodecyl sulfate molecule does not have a significant effect on the mole fraction of IBP in the micelles. On the other hand, the replacement of the compact sulfate head with a bigger one, such as the polyoxyethylene sorbitan head of Tween 20, leads to an increase in $y_{\mathrm{d}}$.

Table 4 provides information about the effect of the type of hydrophobic chain on the value of the mole fraction of IBP in the micelles of the polysorbates (Tweens). The increase in the number of carbon atoms by 4 (from 12 to 16 ) increases the mole fraction of IBP from $y_{\mathrm{d}}=0.52$ to $y_{\mathrm{d}}=0.60$, whereas the further increase to $\mathrm{C} 18$ decreases slightly the IBP mole fraction. Thus, the mole fraction of IBP passes through a maximum when the chain consists of 16 carbon atoms.

The double bond $\mathrm{C}=\mathrm{C}$ in the hydrophobic chain of Tween 80 leads to a significant increase in the mole fraction of IBP: from $y_{\mathrm{d}}=0.57$ for the mixture $\mathrm{C} 16+\mathrm{C} 18$ in Tween 60 , to $y_{\mathrm{d}}=0.64$ for C18 with a double bond (C18:1) in Tween 80. The cis-orientation of the double bond in Tween 80 molecules hinders the close packing in their micellar aggregates and thus facilitates the incorporation of more IBP molecules in the micelles.

Table 4. Mole fraction of IBP in the micelles of nonionic polysorbate (Tween) surfactants.

\begin{tabular}{|l|l|l|}
\hline Trade name & Type of hydrophobic chain & Mole fraction of IBP \\
\hline Tween 20 & Lauric acid (C12) & $0.52 \pm 0.01$ \\
\hline Tween 40 & Palmitic acid (C16) & $0.60 \pm 0.01$ \\
\hline Tween 60 & $\begin{array}{l}\text { Palmitic + stearic acid (C16+ } \\
\text { C18) }\end{array}$ & $0.57 \pm 0.01$ \\
\hline Tween 80 & Oleic acid (C18:1) & $0.64 \pm 0.01$ \\
\hline
\end{tabular}


From thermodynamic viewpoint, the increase of IBP solubility in micellar surfactant solutions can be considered as a process of drug distribution between two phases: (1) water phase and (2) micellar pseudo-phase. At equilibrium, the chemical potential of a drug molecule in the mixed micelles, $\mu_{\text {mic, }}$ is equal to the chemical potential of a drug molecule in the water phase, $\mu_{\mathrm{w}}$. We can calculate the change in the standard chemical potential of the drug molecule, $\Delta \mu_{w / m i c}^{0}$, upon its transfer from water to micelle environment:

$$
\begin{gathered}
\mu_{m i c}=\mu_{w} \quad \text { (at equilibrium) } \\
\mu_{m i c}=\mu_{m i c}^{0}+k T \ln X_{m i c} \\
\mu_{w}=\mu_{w}^{0}+k T \ln X_{w} \\
-\Delta \mu_{w / m i c}^{0}=\mu_{w}^{0}-\mu_{m i c}^{0}=-k T \ln \left(\frac{X_{w}}{X_{m i c}}\right)
\end{gathered}
$$

where $k$ is the Boltzmann constant, $T$ is the absolute temperature, $X_{\text {mic }}$ and $X_{\mathrm{w}}$ are the mole fractions of the drug in the micellar and in the water phase, respectively. The ratio $X_{\text {mic }} / X_{\mathrm{w}}$ is obtained from the experimental solubility data. The values of $\left(\mu_{w}^{0}-\mu_{m i c}^{0}\right)$ are positive, as they express the energy gain associated with the transfer of a poorly-soluble drug molecule from the water environment (unfavorable interactions) into the micelle (more favorable interactions).

Using similar approach, we can calculate the energy associated with the transfer of a molecule from the drug solid phase (crystal) to aqueous solution (in pure water or in surfactant solution). In this case we use the following equation:

$$
-\Delta \mu_{c r / s o l}^{0}=\mu_{c r}^{0}-\mu_{s o l}^{0}=k T \ln X_{s o l}
$$

where $\Delta \mu_{c r / s o l}^{0}$ is the change in the standard chemical potential of the molecule upon transfer from the drug crystal into the solution, and $\mu_{\mathrm{sol}}^{0}$ and $\mu_{c r}^{0}$ are the standard chemical potentials of the drug molecule in the solution and in the solid phase, respectively. $X_{\text {sol }}$ is the mole fraction of the drug dissolved in water or solubilized in the surfactant solution. In contrast to the energy associated with the transfer of a drug molecule from water to the micelle, the values of $\left(\mu_{c r}^{0}-\mu_{n i c}^{0}\right)$ are usually negative, which demonstrates the increase of the energy of a molecule upon drug dissolution from solid phase. This increase of energy is due to the fact that the molecules in the solid phase are surrounded by similar molecules and thus experience very favorable interactions. When placed in contact with molecules having very different properties (e.g. water) the energy of the molecule increases significantly. As the solubility is governed not only by molecular interactions (enthalpic effects), but also by entropic effects, the strong increase of entropy upon dissolution can overcome the unfavorable interactions between the solute molecule and the solvent and thus can result in a measurable solubility. 
The results are summarized in Table 5 and Figure 8. The transfer energy of an IBP molecule from water to micelles is high, almost $13.5 k T$ for all studied surfactants ( $k T$ is the thermal energy). This value is comparable with the results for the solubility of fatty acids in solutions of SLES-1EO and CAPB [36]. As shown in [36], the transfer energy increases with the increase of the fatty acid chain length. The calculated transfer energy of a molecule from water to mixed micelles for palmitic acid (C16) is $14.2 \mathrm{kT}$, which is close to the value calculated for IBP in the current study. This comparison suggests that the cosolubilization process is driven mainly by the hydrophobic attraction between the large hydrophobic fragment of IBP molecule and the surfactant tails. In addition, the polar carboxyl group in the IBP molecule is expected to be oriented towards the micelle surface and could provide additional attraction, via hydrogen bonds and/or dipole-dipole interaction, with the surfactant head-groups. A molecular modelling of the structure of the mixed micelles (which is beyond the scope of the present study) could provide deeper insight about the molecular arrangement and the specific interactions governing the comicellization process.

Let us now examine the results for the transfer of an IBP molecule from a drug crystal into water or into a surfactant solution. The transfer to the water environment is highly unfavorable $(-14.3 k T)$ and in a good agreement with the observed very low solubility of the IBP in water. On the other hand, all values for the transfer energy of IBP from a crystal to the micellar solutions vary between -0.5 and $-1.0 \mathrm{kT}$. These values are of the order of the thermal energy and are, thus, in a good agreement with the observed high solubility of IBP in the micellar solutions.

Table 5. Transfer energy of an IBP molecule from a crystal to a mixed micelle and from water to a mixed micelle. The transfer energy for a molecule IBP from a crystal to water is $-14.3 k T$. The energies are calculated using eqs. (7) and (8).

\begin{tabular}{|l|l|l|}
\hline Surfactant & Crystal $\rightarrow$ micelle, $-\Delta \mu_{c r / s o l}^{0} / k T$ & Water $\rightarrow$ micelle, $-\Delta \mu_{w / m i c}^{0} / k T$ \\
\hline Tween 20 & $-0.66 \pm 0.02$ & $13.65 \pm 0.02$ \\
\hline Tween 40 & $-0.52 \pm 0.01$ & $13.79 \pm 0.01$ \\
\hline Tween 60 & $-0.56 \pm 0.01$ & $13.75 \pm 0.01$ \\
\hline Tween 80 & $-0.54 \pm 0.01$ & $13.86 \pm 0.01$ \\
\hline SDS & $-0.86 \pm 0.01$ & $13.45 \pm 0.01$ \\
\hline SDS + NaCl & -1.04 & 13.27 \\
\hline SLES-3EO & -0.94 & 13.37 \\
\hline SLES-3EO $+\mathrm{NaCl}$ & -0.84 & 13.46 \\
\hline
\end{tabular}




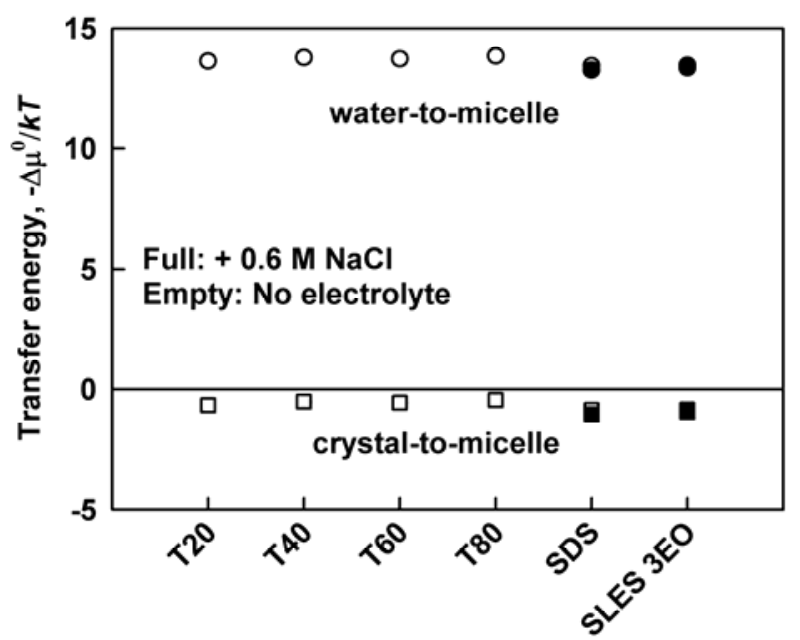

Figure 8. Transfer energy of an IBP molecule from an IBP crystal into a mixed micelle (squares) and from water to a mixed micelle (circles). The results are for surfactant solutions in the absence (empty symbols) and in the presence of $0.6 \mathrm{M} \mathrm{NaCl}$ (full symbols). The transfer energy for an IBP molecule from a crystal to water is $-14.3 k T$ ( $k T$ is the thermal energy). The energies are calculated using eqs. (7) and (8).

\section{CONCLUSIONS}

We performed an experimental study on the solubility of IBP in aqueous surfactant solutions. We studied a total of 6 surfactants: four nonionic (polysorbate Tween 20, 40, 60 and 80) and two anionic (SDS and SLES-3EO). The mechanism of improved solubility of IBP and the strength of drug-surfactant interactions was assessed by thermodynamic treatment of the solubility data.

The main conclusions of the current study can be summarized as follows:

1. At a constant concentration of $0.5 \mathrm{wt} \%$, the anionic surfactants SDS and SLES-3EO and the nonionic Tween 80 improve strongly the IBP solubility in the aqueous phase - by a factor of 200 .

2. The mechanism of solubility enhancement is that IBP forms mixed micelles with all studied surfactants. This explanation is proved by the exceptionally high molar fraction of IBP in the micelles, $y_{\mathrm{d}}$ $=0.4$ to 0.6 for all surfactant solutions studied, and by the very high rate of the solubilization process. The energy gain upon transfer of an IBP molecule from the water environment into the mixed micelles is very high, 13 to $14 \mathrm{kT}$ units for all surfactant solutions studied.

3. The highest mole fraction of the drug in the mixed micelles is obtained with Tween 80 : almost $2 / 3$ of all molecules in the mixed micelles are of IBP $\left(y_{\mathrm{d}}=0.64\right)$. The increase of the number of carbon atoms in the hydrophobic chain of the nonionic polysorbate surfactants from 12 to 16 atoms (Tween 20 to Tween 60) increases the molar fraction of IBP in the micelles. Higher drug molar fraction is also obtained when the hydrophobic chain of the polysorbate surfactants contains a double bond (Tween 80). 


\section{ACKNOWLEDGMENTS}

The author gratefully acknowledge Mrs. Mariana Paraskova for technical editing of the manuscript.

\section{FUNDING}

This research did not receive any specific grant from funding agencies in the public, commercial, or notfor-profit sectors.

\section{REFERENCES}

1. D. Thompson, M.V. Chaubal, Cyclodextrins (CDS) - excipients by definition, drug delivery systems by function (part I: injectable applications), Drug Deliv. Technol. 2 (2000) 34-38.

2. C.O. Rangel-Yagui, J.A. Pessoa, L.C. Tavares, Micellar solubilization of drugs. J. Pharm. Pharm. Sci. 8 (2005) 147-163.

3. A.T. Florence, Technique of Solubilization of Drugs, Marcel Dekker, New York, 1981.

4. J. Swarbrick, Solubilized systems in pharmacy, J. Pharm. Sci. 54 (1965) 1229-1237, DOI: 10.1002/jps.2600540902.

5. V.P. Torchilin, Micellar nanocarriers: Pharmaceutical perspectives, Pharm. Res. 24 (2007) 1-16, DOI: $10.1007 / \mathrm{s} 11095-006-9132-0$.

6. D. Attwood, A.T. Florence, Surfactant Systems: Their Chemistry, Pharmacy, and Biology, Chapman and Hall, London, New York, 1983.

7. M.J. Rosen, M.J. Surfactants and Interfacial Phenomena, third ed., John Wiley \& Sons, Inc., New Jersey, 2004.

8. A.R. Tehrani-Bagha, K. Holmberg, Solubilization of hydrophobic dyes in surfactant solutions. Materials 6 (2013) 580-608, DOI:10.3390/ma6020580.

9. M.A. Rub, N. Azum, A.M. Asiri, Interaction of cationic amphiphilic drug nortriptyline hydrochloride with TX-100 in aqueous and urea solutions and the studies of physicochemical parameters of the mixed micelles, J. Mol. Liq. 218 (2016) 595-603, DOI: 10.1016/j.molliq.2016.02.049.

10. D. Kumar, M.A. Rub, Aggregation behavior of amphiphilic drug promazine hydrochloride and sodium dodecylbenzenesulfonate mixtures under the influence of $\mathrm{NaCl} /$ urea at various concentration and temperatures, J. Phys. Org. Chem. 29 (2016) 394-405, DOI: 10.1002/poc.3546.

11. S.-H. Park, H.-K. Choi, The effects of surfactants on the dissolution profiles of poorly water-soluble acidic drugs, Int. J. Pharm. 321 (2006) 35-41, DOI: 10.1016/j.ijpharm.2006.05.004.

12. S. Di Maio, R. Carrier, R. Gastrointestinal contents in fasted state and post-lipid ingestion: In vivo measurements and in vitro models for studying oral drug delivery, J. Control. Release 151 (2011) 110-122, DOI: 10.1016/j.jconrel.2010.11.034 .

13. A.L. Daugherty, R.J. Mrsny, Regulation of the intestinal epithelial paracellular barrier, Pharm. Sci. Technol. Today 2 (1999) 281-287, DOI: 10.1016/S1461-5347(99)00170-4 . 
14. M. Yazdanian, K. Briggs, C. Jankovsky, A. Hawi, The "High Solubility" definition of the current FDA guidance on biopharmaceutical classification system may be too strict for acidic drugs, Pharm. Res. 21 (2004) 293-299.

15. G.L. Amidon, H. Lennernas, V.P. Shah, J.R. Crison, A theoretical basis for a biopharmaceutic drug classification: The correlation of in vitro drug product dissolution and in vivo bioavailability, Pharm. Res. 12 (1995) 413-420.

16. T. Takagi, C. Ramachandran, M. Bermejo, S. Yamashita, L.X. Yu, G.L. Amidon, A provisional biopharmaceutical classification of the top 200 oral drug products in the United States, Great Britain, Spain, and Japan, Mol. Pharm. 3 (2006) 631-643, DOI: 10.1021/mp0600182.

17. C. Alvarez, I. Núñez, J.J. Torrado, J. Gordon, H. Potthast, A. García-Arieta, Investigation on the possibility of biowaivers for Ibuprofen, J. Pharm. Sci. 100 (2011) 2343-2349, DOI: 10.1002/jps.22472.

18. E. Bulut, M. Dilek, Development and characterization of $\mathrm{pH}$-sensitive locust bean gum-alginate microspheres for controlled release of ibuprofen, J. Drug Delivery Sci. Techn. 24 (2014) 613-619, DOI:10.1016/S1773-2247(14)50127-X.

19. T. Jambwa, A. Viljoen, J. Hamman, Matrix forming excipients from natural origin for controlled release matrix type tablets, J. Drug Delivery Sci. Techn. 21 (2011) 433-439, DOI: 10.1016/S17732247(11)50069-3.

20. M. Moneghini, N. De Zordi, M. Grassi, G. Zingone, Sustained-release solid dispersions of ibuprofen prepared by microwave irradiation, J. Drug Delivery Sci. Techn. 18 (2008) 327-333, DOI: 10.1016/S1773-2247(08)50064-5.

21. F. Lagarce, O. Thomas, Lipid nanocapsules: A nanocarrier suitable for scale-up process, J. Drug Delivery Sci. Techn. 23 (2013) 555-559, DOI: 10.1016/S1773-2247(13)50084-0.

22. A. Tan, N.G. Eskandar, S. Rao, C.A. Prestidge, First in man bioavailability and tolerability studies of a silica-lipid hybrid (Lipoceramic) formulation: A Phase I study with ibuprofen, Drug Delivery Trans. Res. 4 (2014) 212-221, DOI: 10.1007/s13346-013-0172-9 .

23. Y. Zhai, G. Zhai, Advances in lipid-based colloid systems as drug carrier for topic delivery, J. Controlled Release 193 (2014) 90-99, DOI: 10.1016/j.jconrel.2014.05.054.

24. I.U. Khan, N.M. Ranjha, H.Q. Mehmood, Development of ethylcellulose-polyethylene glycol and ethylcellulose- polyvinyl pyrrolidone blend oral microspheres of ibuprofen, J. Drug Delivery Sci. Techn. 20 (2010) 439-444, DOI: 10.1016/S1773-2247(10)50076-5.

25. S. Plakkot, M. De Matas, P. York, M. Saunders, B. Sulaiman, Comminution of ibuprofen to produce nano-particles for rapid dissolution, Int. J. Pharm. 415 (2011) 307-314, DOI: 10.1016/j.ijpharm.2011.06.002.

26. C. Marianecci, F. Rinaldi, L.D. Marzio, A. Ciogli, S. Esposito, M. Carafa, Polysorbate 20 vesicles as multi-drug carriers: In vitro preliminary evaluations, Lett. Drug Design Discovery 10 (2015) 212 218. 
27. B.C. Stephenson, C.O. Rangel-Yagui, A. Pessoa, L.C. Tavares, K. Beers, D. Blankschtein, Experimental and theoretical investigation of the micellar-assisted solubilization of ibuprofen in aqueous media, Langmuir 22 (2006) 1514-1525, DOI: 10.1021/la052530k .

28. Z. Kokot, H. Zmidzinska, Solubility and Dissolution Rate of Ibuprofen in Ionic and Non-ionic Micellar Systems, Acta Poloniae Pharm. - Drug Res. 58 (2001) 117-120.

29. S.-H. Park, H.-K. Choi, The effects of surfactants on the dissolution profiles of poorly water-soluble acidic drugs, Int. J. Pharmm. 32 (2006) 35-41.

30. Z. Vinarov, D. Radeva, S. Tcholakova, N. Denkov, Effect of surfactant type on the solubilization of poorly water-soluble drugs, Article in preparation.

31. M.J. Schick, Nonionic Surfactants, Marcel Dekker, New York, 1967.

32. S.J. Rehfeld, Adsorption of Sodium Dodecyl Sulfate at Various Hydrocarbon-Water Interfaces, J. Phys. Chem. 71 (1967) 738-745, DOI: 10.1021/j100862a039.

33. S.E. Anachkov, K.D. Danov, E.S. Basheva, P.A. Kralchevsky, K.P. Ananthapadmanabhan, Determination of the aggregation number and charge of ionic surfactant micelles from the stepwise thinning of foam films, Adv. Colloid Interface Sci. 183-184 (2012) 55-67, DOI: 10.1016/j.cis.2012.08.003.

34. A. Fini, M. Laus, I. Orienti, V. Zecchi, Dissolution and partition thermodynamic functions of some nonsteroidal anti-inflammatory drugs, J. Pharm. Sci. 75 (1986) 23-25, DOI: 10.1002/jps.2600750106.

35. V. Gaikar, V. Latha, Hydrotropic Properties of Sodium Salt of Ibuprofen, Drug Dev. Ind. Pharm. 23 (1997) 309-312, DOI: 10.3109/03639049709149808.

36. S.S. Tzocheva, P.A. Kralchevsky, K.D. Danov, G.S. Georgieva, A.J; Post, K.P. Ananthapadmanabhan, Solubility limits and phase diagrams for fatty acids in anionic (SLES) and zwitterionic (CAPB) micellar surfactant solutions, J. Colloid Interface Sci. 369 (2012) 274-286, DOI: 10.1016/j.jcis.2011.12.036.

37. P.D. Todorov, P.A. Kralchevsky, N.D. Denkov, G. Broze, A. Mehreteab, Kinetics of Solubilization of n-Decane and Benzene by Micellar Solutions of Sodium Dodecyl Sulfate, J. Colloid Interface Sci. 245 (2002) 371-382, DOI: 10.1006/jcis.2001.8031

38. M.A. Rub, F. Khan, M.S. Sheikh, N. Azum, A.M. Asiri, Tensiometric, fluorescence and 1H NMR study of mixed micellization of non-steroidal anti-inflammatory drug sodium salt of ibuprofen in the presence of non-ionic surfactant in aqueous/urea solutions, J. Chem. Therm. 96 (2016) 196-207, DOI: 10.1016/j.jct.2016.01.001.

39. S. Das, S. Mondal, S. Ghosh, Physicochemical Studies on the Micellization of Cationic, Anionic, and Nonionic Surfactants in Water-Polar Organic Solvent Mixtures, J. Chem. Eng. Data 58 (2013) 25862595. DOI: $10.1021 / \mathrm{je} 4004788$. 